\title{
Direct and indirect effects of sociocultural influences on disordered eating among Malaysian male and female university students : a mediation analysis of psychological distress.
}

\begin{abstract}
This study aimed to examine the role of psychological distress in the relationships between sociocultural influences (social pressure to be thin and weight teasing) and disordered eating. Data were collected from 584 university students (59.4\% females and $40.6 \%$ males), aged 18-24 years old $(M=20.6, S D=1.4)$, selected from four universities in the Klang Valley, Malaysia. Participants completed four standardized questionnaires which measured social pressure to be thin, weight-related teasing, psychological distress and disordered eating. A good fit structural equation modeling (SEM) model was developed for both sexes. For males, the SEM model revealed that sociocultural influences showed an indirect effect on disordered eating through psychological distress. For females, the model showed an indirect effect of sociocultural influences on disordered eating through psychological distress, as well as a direct effect of sociocultural influences on disordered eating. In conclusion, psychological distress mediated the relationships between sociocultural influences and disordered eating in both males and females. Our results suggest that disordered eating intervention programs on reducing psychological distress in university students may be beneficial.
\end{abstract}

Keyword: Disordered eating; Psychological distress; Weight-related teasing; Pressure to be thin; Direct and indirect effects; Structural equation model. 